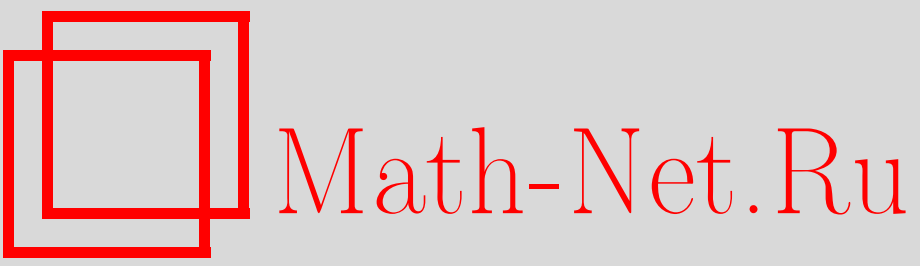

V. Cammarota, E. Orsingher, Angular processes related to Cauchy random walks, Теория вероятн. и ее примен., 2010, том 55, выпуск 3, 489-506

DOI: https://doi.org/10.4213/tvp4238

Использование Общероссийского математического портала Math-Net.Ru подразумевает, что вы прочитали и согласны с пользовательским соглашением

http://www.mathnet.ru/rus/agreement

Параметры загрузки:

IP: 3.89 .197 .203

26 апреля 2023 г., 10:52:59 
ТЕОРИЯ ВЕРОЯТНОСТЕЙ

Том 55

И ЕЕ ПРИМЕНЕНИЯ

Выпуск 3

2010

(C) 2010 г. CAMMAROTA V.* , ORSINGHER E.*

\title{
ANGULAR PROCESSES RELATED TO CAUCHY RANDOM WALKS
}

\begin{abstract}
В статье изучается угловой процесс, связанный со случайным блужданиями в евклидовом и неевклидовом пространстве, шаги которых имеют распределение Коши.

Это приводит к различного рода нелинейным преобразованиям случайных величин с распределением Коши, сохраняющим плотность Коши. Мы приводим явный вид этих распределений для всевозможных комбинаций параметров масштаба и сдвига.

Анализируются непрерывные дроби, содержащие случайные величины с распределением Коши. Показано, что на $n$-м этапе случайные величины все еще имеют распределение Коши с параметрами, связанными с числами Фибоначчи. Это позволяет нам доказать, что последовательность сходится по распределению к золотому сечению.
\end{abstract}

Ключевые слова и фразы: гиперболическая тригонометрия, закон арксинуса, непрерывные дроби, числа Фибоначчи, нелинейные преобразования случайных величин.

1. Introduction. We consider a particle starting from the origin $O$ of $\mathbf{R}^{2}$ which takes initially a horizontal step of length 1 and a vertical one, say $\mathbf{C}_{1}$, with a standard Cauchy distribution. It reaches therefore the position $\left(1, \mathbf{C}_{1}\right)$. The line $l_{1}$ joining the origin with $\left(1, \mathbf{C}_{1}\right)$ forms a random angle $\Theta_{1}$ with the horizontal axis (see Figure 1).

On $l_{1}$ the traveller repeats the same movement with a step of unit length (either forward or backward) along $l_{1}$ and a standard Cauchy distributed step, say $\mathbf{C}_{2}$, on the line orthogonal to $l_{1}$. The right triangle obtained with the last two displacements has a hypotenuse belonging to the line $l_{2}$ with random inclination $\Theta_{2}$ on $l_{1}$.

*Dipartimento di Statistica, Probabilità e Statistiche applicate, University of Rome «La Sapienza», P.le Aldo Moro 5, 00185 Rome, Italy; e-mail: valentina.cammarota@uniroma1.it.; enzo.orsingher@uniroma1.it 

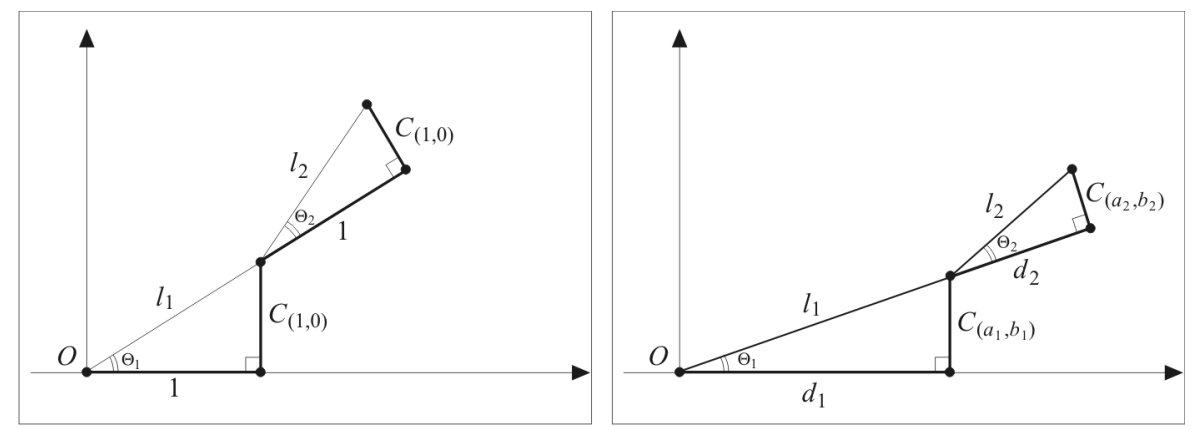

Fig. 1. The angular process in the Euclidean plane. By $C_{\left(a_{j}, b_{j}\right)}$ we indicate the $j$-th random displacement with Cauchy distribution possessing scale parameter $a_{j}$ and location parameter $b_{j}$

After $n$ steps the sequence of random angles $\Theta_{1}, \ldots, \Theta_{n}$ describes the rotation of the moving particle around the starting point, their partial sums describe an angular random walk which can be written as

$$
S_{n}=\Theta_{1}+\cdots+\Theta_{n}=\sum_{j=1}^{n} \operatorname{arctg} \mathbf{C}_{j}
$$

where $\mathbf{C}_{j}$ are independent standard Cauchy random variables. If the random steps of the planar random walk above were independent Cauchy random variables with scale parameter $a_{j}$ and location parameter $b_{j}$, then the process (1.1) must be a little bit modified and rewritten as

$$
S_{n}=\Theta_{1}+\cdots+\Theta_{n}=\sum_{j=1}^{n} \operatorname{arctg} C_{j},
$$

where $C_{j} \sim C_{\left(a_{j}, b_{j}\right)}$. The model (1.2) can be extended also to the case where the first step has length $d_{j}$ and the second one is Cauchy distributed with scale parameter $a_{j}$ and location parameter $b_{j}$ (see Figure 1), then

$$
\operatorname{tg} \Theta_{j}=C_{\left(a_{j} / d_{j}, b_{j} / d_{j}\right)} .
$$

The same random walk can be generated if the two orthogonal steps, at each displacement, are represented by two independent Gaussian random variables $X_{j}$ and $Y_{j}$. In this case, for each right triangle, we can write

$$
\operatorname{tg} \Theta_{j}=\frac{X_{j}}{Y_{j}}
$$

If $X_{j}$ and $Y_{j}$ are two standard independent Gaussian random variables then $\operatorname{tg} \Theta_{j}=X_{j} / Y_{j}$ possesses standard Cauchy distribution and we get the model in (1.1). The model (1.2) can be obtained by considering orthogonal Gaussian steps with different variances and in this case the scale parameter of the random variables $C_{j}$ is the ratio $a_{j}=\sigma_{j}^{Y} / \sigma_{j}^{X}$. 
The model (1.1) describing the angular random process has a hyperbolic counterpart. We consider a particle starting from the origin $O$ of the Poincare half-plane $\mathbf{H}_{+}^{2}=\{(x, y): y>0\}$. At the $j$-th displacement, $j=1,2, \ldots$, the particle makes two steps of random hyperbolic length $\eta_{j}$ and $\widehat{\eta}_{j}$ on two orthogonal geodesic lines. The $j$-th displacement leads to a right triangle $T_{j}$ with sides of length $\eta_{j}$ and $\widehat{\eta}_{j}$ and random acute angles $\Theta_{j}$ and $\widehat{\Theta}_{j}$. In each triangle $T_{j}$ the first step is taken on the extension of the hypotenuse of the triangle $T_{j-1}$ (see Figure 2). From hyperbolic trigonometry (for basic results on hyperbolic geometry see, for example, [5]) we have that

$$
\sin \Theta_{j}=\frac{\operatorname{sh} \widehat{\eta}_{j}}{\sqrt{\operatorname{ch}^{2} \eta_{j} \operatorname{ch}^{2} \widehat{\eta}_{j}-1}}, \quad \cos \Theta_{j}=\frac{\operatorname{sh} \eta_{j} \operatorname{ch} \widehat{\eta}_{j}}{\sqrt{\operatorname{ch}^{2} \eta_{j} \operatorname{ch}^{2} \widehat{\eta}_{j}-1}}
$$

From the above expressions we have that

$$
\operatorname{tg} \Theta_{j}=\frac{\operatorname{th} \widehat{\eta}_{j}}{\operatorname{sh} \eta_{j}} .
$$

If we take independent random hyperbolic displacements $\eta_{j}$ and $\widehat{\eta}_{j}$ such that the random variables $E_{j}=\frac{\operatorname{th} \widehat{\eta}_{j}}{\operatorname{sh} \eta_{j}}$ are standard Cauchy distributed, then $\Theta_{j}=\operatorname{arctg} \mathbf{C}_{j}$. If the triangles $T_{j}$ were isosceles, then $\operatorname{tg} \Theta_{j}=1 / \operatorname{ch} \eta_{j}$ and $\Theta_{j} \in[-\pi / 4, \pi / 4]$ so that in this case the Cauchy distribution cannot be attributed to $\operatorname{tg} \Theta_{j}$.

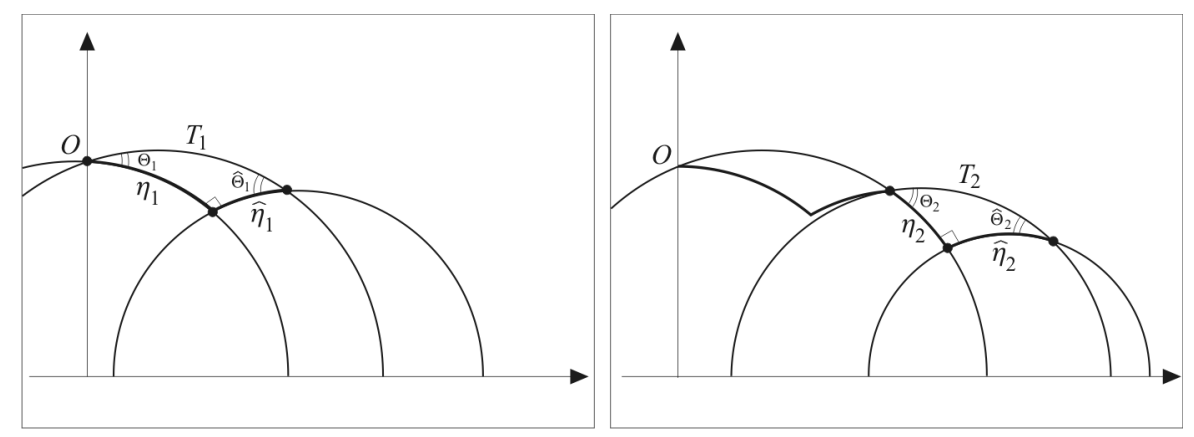

Fig. 2. The angular random process in the Poincaré half-plane

In the model described here the random steps (and therefore the random angular windings $\Theta_{j}$ ) are independent. If we consider the model of papers [2] and [3], where the displacements are taken orthogonally to the geodesic lines joining the origin $O$ of $\mathbf{H}_{+}^{2}$ with the positions occupied at deviation instants, the angular displacements $\Theta_{j}$ must be such that

$$
\sin \Theta_{j}=\frac{\operatorname{sh} \eta_{j}}{\sqrt{1+\prod_{r=1}^{j} \operatorname{ch}^{2} \eta_{r}}}=\operatorname{sh} \eta_{j} \cos \left(\operatorname{arctg} \prod_{r=1}^{j} \operatorname{ch}^{2} \eta_{r}\right)
$$


and therefore involve dependent random variables.

For the area of the random hyperbolic triangle $T_{j}$ we note that

$$
\begin{aligned}
\operatorname{area}\left(T_{j}\right) & =\frac{\pi}{2}-\Theta_{j}-\widehat{\Theta}_{j}=\frac{\pi}{2}-\left[\operatorname{arctg}\left(\frac{\operatorname{th} \widehat{\eta}_{j}}{\operatorname{sh} \eta_{j}}\right)+\operatorname{arctg}\left(\frac{\operatorname{th} \eta_{j}}{\operatorname{sh} \widehat{\eta}_{j}}\right)\right] \\
& =\frac{\pi}{2}-\operatorname{arctg}\left(\frac{\operatorname{coth} \eta_{j}}{\operatorname{sh} \widehat{\eta}_{j}}+\frac{\operatorname{coth} \widehat{\eta}_{j}}{\operatorname{sh} \eta_{j}}\right)=\operatorname{arcctg}\left(\frac{\operatorname{coth} \eta_{j}}{\operatorname{sh} \widehat{\eta}_{j}}+\frac{\operatorname{coth} \widehat{\eta}_{j}}{\operatorname{sh} \eta_{j}}\right) .
\end{aligned}
$$

Since each acute angle inside $T_{j}$ is linked to both sides of the triangle, the analysis of the random process $A_{n}=\sum_{j=1}^{n}$ area $\left(T_{j}\right)$ is much more complicated and we drop it.

Let $C_{j} \sim C_{\left(a_{j}, b_{j}\right)}, j=1,2, \ldots$, be independent Cauchy random variables, where $a_{j}$ is the scale parameter and $b_{j}$ is the location parameter. In the study of the angular random walk (1.1) and (1.2) we must analyze the distribution of the following nonlinear transformations of Cauchy random variables:

$$
U=\frac{C_{1}+C_{2}}{1-C_{1} C_{2}}
$$

since

$$
\operatorname{arctg} C_{1}+\operatorname{arctg} C_{2}=\operatorname{arctg} \frac{C_{1}+C_{2}}{1-C_{1} C_{2}} .
$$

Since the 1960 a wide class of nonlinear transformations of Cauchy random variables has been considered. Williams [10], Knight [6] and Letac [7] proved that transformations of the form

$$
\varepsilon f(x)=k x+\alpha-\sum_{i=1}^{n} \frac{\mu_{i}}{x-\gamma_{i}},
$$

where $\varepsilon= \pm 1, \alpha, \gamma_{i} \in \mathbf{R}$ and $k, \mu_{i} \geqslant 0$, preserve the Cauchy distribution.

In particular, in [10] the following characterization for Cauchy random variables is proved. The random variable $X$ is a standard Cauchy if and only if $(1+b X) /(b-X)$ is a standard Cauchy for some constant $b$ which is not the tangent of a rational multiple of $\pi$.

Knight [6] asserts that a random variable $X$ is of Cauchy type if and only if the random variable $(a X+b) /(c X+d)$ is still of Cauchy type, whenever $a d-b c \neq 0$.

Our problem is more strictly related to the results obtained by Pitman and Williams [8]. They proved that the standard Cauchy distribution is preserved under certain types of transformations represented by meromorphic functions whose poles are all real and simple. As a corollary they obtained that, if $P$ and $Q$ are two independent random variables uniformly distributed in $(0, \pi)$, then the random variables $X=\operatorname{tg} P$ and $Y=\operatorname{tg} Q$ are standard Cauchy and

$$
\operatorname{tg}(P+Q)=\frac{X+Y}{1-X Y} \stackrel{\text { law }}{=} X
$$


We will show that the random variable (1.3) is endowed with Cauchy distribution in a much more general situation, namely when the random variables $C_{j}, j=1,2$, have nonzero location parameters $b_{j}$ and scale parameters $a_{j}$. The scale and location parameters of $U$ depend on both parameters $a_{j}$ and $b_{j}$ suitably combined.

In particular, if $b_{1}=b_{2}=0$ and $a_{1}=a_{2}=1$, then $U$ is still distributed as a standard Cauchy distribution and therefore in (1.1) we have that

$$
S_{n} \stackrel{\operatorname{law}}{=} \operatorname{arctg} \mathbf{C} .
$$

Since also $1 / \mathbf{C}$ is a standard Cauchy (for basic properties of Cauchy random variables see, for example, [9, p. 105], from (1.3), a number of related random variables preserving the form of the Cauchy distribution can be considered. For example, the random variables

$$
Z_{1}=\frac{\mathbf{C}_{1} \mathbf{C}_{2}+1}{\mathbf{C}_{1}-\mathbf{C}_{2}}, \quad Z_{2}=\frac{1-\mathbf{C}_{1} \mathbf{C}_{2}}{\mathbf{C}_{1}+\mathbf{C}_{2}}, \quad Z_{3}=\frac{\mathbf{C}_{1}+\mathbf{C}_{2}}{\mathbf{C}_{1} \mathbf{C}_{2}-1}
$$

also possess standard Cauchy distribution.

If $b_{1}=b_{2}=0$ and the scale parameters $a_{1}, a_{2}$ are different, then (1.3) still preserves the Cauchy distribution but with scale parameter equal to $\left(a_{1}+a_{2}\right) /\left(1+a_{1} a_{2}\right)$ and location parameter equal to zero. This can be grasped by means of the following relationship:

$$
\operatorname{arctg} C_{1}+\operatorname{arctg} C_{2} \stackrel{\operatorname{law}}{=} \operatorname{arctg}\left\{\frac{a_{1}+a_{2}}{1+a_{1} a_{2}} \mathbf{C}\right\}
$$

where $C_{j} \sim C_{\left(a_{j}, 0\right)}$. Result (1.4) is illustrated in Figure 3 .

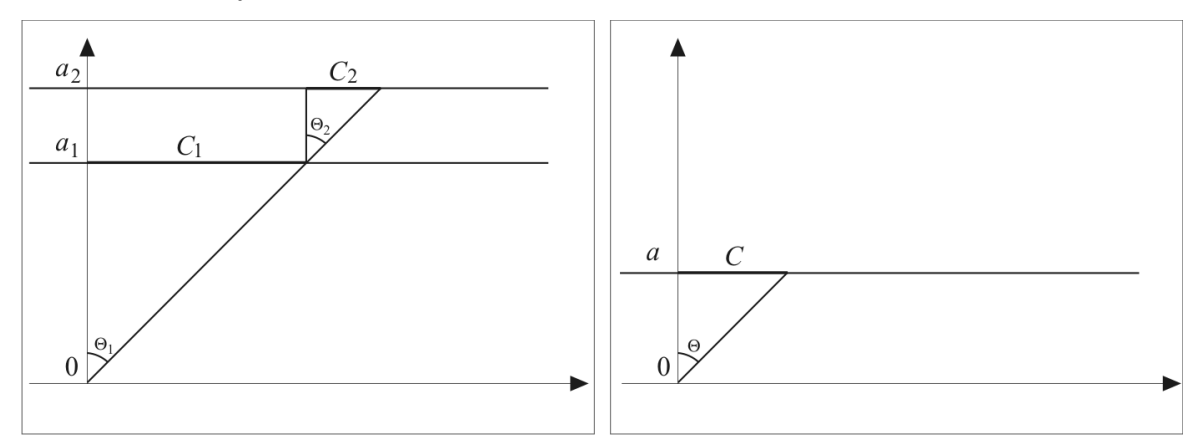

Fig. 3. The figure shows that shooting a ray with inclination $\Theta_{1}$, uniformly distributed, against the line at distance $a_{1}$ and then shooting a ray with a uniformly distributed angle $\Theta_{2}$ on the line at distance $a_{2}$ is equivalent to shooting on the barrier at the distance $a=\left(a_{1}+a_{2}\right) /\left(1+a_{1} a_{2}\right)$ with a uniformly distributed angle $\Theta$

By iterating the process (1.4) we arrive at the formula

$$
\sum_{j=1}^{3} \operatorname{arctg} C_{j} \stackrel{\operatorname{law}}{=} \operatorname{arctg}\left\{\frac{\sum_{j=1}^{3} a_{j}+a_{1} a_{2} a_{3}}{1+\sum_{i \neq j} a_{i} a_{j}} \mathbf{C}\right\}
$$


which gives an insight into further extensions of the process outlined above.

Much more complicated are the cases, where the location parameters of the Cauchy distributions are different from zero. For the special case, where $C_{1}$ and $C_{2}$ are independent Cauchy such that $C_{1} \sim C_{(1, b)}$ and $C_{2} \sim C_{(1, b)}$, the random variable (1.3) still possesses Cauchy density with scale parameter $\left(2 b^{2}+4\right) /\left(b^{4}+4\right)$ and location parameter $2 b^{3} /\left(b^{4}+4\right)$.

We have obtained the general distribution of (1.3), where $C_{1}$ and $C_{2}$ are independent Cauchy such that $C_{1} \sim C_{\left(a_{1}, b_{1}\right)}$ and $C_{2} \sim C_{\left(a_{2}, b_{2}\right)}$, and also the distribution of

$$
U=\frac{\gamma C_{1}+\delta C_{2}}{\alpha-\beta C_{1} C_{2}}
$$

for arbitrary positive real numbers $\beta, \gamma, \delta$ and $\alpha+\beta \neq 0$. In particular, if $C_{1}$ and $C_{2}$ are independent standard Cauchy random variables, then $U$ is Cauchy with scale parameter equal to $|(\gamma+\delta) /(\alpha+\beta)|$ and location parameter equal to zero.

In the last section we examine continued fractions involving Cauchy random variables. In particular, we have studied

$$
V_{n}=\frac{1}{1+\frac{1}{1+\frac{1}{1+\mathrm{C}}}}
$$

and

$$
U_{n}=\frac{1}{1+\frac{1}{1+\frac{1}{1+\mathrm{C}^{2}}}}
$$

which generalize the random variables $V_{1}=1 /(1+\mathbf{C})$ and $U_{1}=1 /\left(1+\mathbf{C}^{2}\right)$. Continued fractions involving random variables have been analyzed in [4] and more recently in [1]. The random variable $U_{1}$ has the arcsine distribution in $[0,1]$, while $U_{t}=t U_{1}$, with $t>0$, has distribution

$$
\mathbf{P}\left\{U_{t} \in \mathrm{d} s\right\}=\frac{\mathrm{d} s}{\pi \sqrt{s(t-s)}}, \quad 0<s<t .
$$

For each $n \geqslant 1$, the random variables $V_{n}$ are Cauchy distributed with scale parameter $a_{n}$ and location parameter $b_{n}$ that can be expressed in terms of Fibonacci numbers. This permits us to prove the monotonicity of $a_{n}$ and $b_{n}$ and that $\lim _{n \rightarrow \infty} a_{n}=0$ and $\lim _{n \rightarrow \infty} b_{n}=\varphi-1$, where $\varphi=(1+\sqrt{5}) / 2$ is the golden ratio. Finally we obtain that the sequences of random variables $1+V_{n}$ and $1+U_{n}, n \geqslant 1$, converge in distribution to the number $\varphi=(1+\sqrt{5}) / 2$. This should be expected, since it has the infinite fractional expansion

$$
\frac{1+\sqrt{5}}{2}=1+\frac{1}{1+\frac{1}{1+\cdots}}
$$

which is related to (1.5) and (1.6). 
2. Centered Cauchy random variables. In this section we study the distribution of the following random variable:

$$
U=\frac{\gamma \mathbf{C}_{1}+\delta \mathbf{C}_{2}}{\alpha-\beta \mathbf{C}_{1} \mathbf{C}_{2}}
$$

where $\mathbf{C}_{1}$ and $\mathbf{C}_{2}$ are independent, standard Cauchy. We assume, without restrictions, that $\beta, \gamma, \delta$ are nonnegative real numbers all different from zero (because of the symmetry of $\left.\mathbf{C}_{j}, j=1,2\right)$ and $\alpha+\beta \neq 0$. In the following theorem we prove that $U$ is still Cauchy distributed.

Theorem 2.1. The random variable $U$ in (2.1) possesses Cauchy distribution with scale parameter equal to $|(\gamma+\delta) /(\alpha+\beta)|$ and location parameter equal to zero. We can also restate the result in symbols as

$$
U \stackrel{\text { law }}{=} \frac{\gamma+\delta}{\alpha+\beta} \mathbf{C}
$$

P r o o f. The density of the random variable $U$ can be obtained by means of the transformation

$$
u=\frac{\gamma x+\delta y}{\alpha-\beta x y}, \quad v=y
$$

with $(x, y) \in \mathbf{R}^{2}$ and Jacobian equal to

$$
|J|=\frac{\alpha \gamma+\delta \beta v^{2}}{(\gamma+\beta u v)^{2}}
$$

The joint density $g=g(u, v)$ reads

$$
\begin{aligned}
g(u, v) & =\frac{1}{\pi^{2}\left(1+v^{2}\right)} \frac{1}{1+((\alpha u-\delta v) /(\gamma+\beta u v))^{2}} \frac{\alpha \gamma+\delta \beta v^{2}}{(\gamma+\beta u v)^{2}} \\
& =\frac{1}{\pi^{2}\left(1+v^{2}\right)} \frac{\alpha \gamma+\delta \beta v^{2}}{v^{2}\left(\beta^{2} u^{2}+\delta^{2}\right)+2 u v(\beta \gamma-\delta \alpha)+\alpha^{2} u^{2}+\gamma^{2}},
\end{aligned}
$$

where $(u, v) \in \mathbf{R}^{2}$, and must be integrated with respect to $v$ in order to obtain the distribution of $U$. Therefore

$$
\begin{aligned}
& \mathbf{P}\left\{\frac{\gamma \mathbf{C}_{1}+\delta \mathbf{C}_{2}}{\alpha-\beta \mathbf{C}_{1} \mathbf{C}_{2}} \in \mathrm{d} u\right\} \\
& =\frac{\mathrm{d} u}{\pi^{2}} \int_{-\infty}^{\infty}\left[\frac{1}{1+v^{2}} \frac{\alpha \gamma+\delta \beta v^{2}}{v^{2}\left(u^{2} \beta^{2}+\delta^{2}\right)+2 v u(\beta \gamma-\delta \alpha)+u^{2} \alpha^{2}+\gamma^{2}}\right] \mathrm{d} v \\
& =\frac{\mathrm{d} u}{\pi^{2}} \int_{-\infty}^{\infty}\left[\frac{A v+B}{1+v^{2}}+\frac{C v+D}{v^{2}\left(u^{2} \beta^{2}+\delta^{2}\right)+2 v u(\beta \gamma-\delta \alpha)+u^{2} \alpha^{2}+\gamma^{2}}\right] \mathrm{d} v
\end{aligned}
$$


where

$$
\begin{aligned}
A & =\frac{2 u(\beta \gamma-\alpha \delta)(\beta \delta-\alpha \gamma)}{\left[u^{2}(\alpha-\beta)^{2}+(\gamma-\delta)^{2}\right]\left[u^{2}(\alpha+\beta)^{2}+(\gamma+\delta)^{2}\right]} \\
B & =\frac{(\alpha \gamma-\beta \delta)\left[\left(\gamma^{2}-\delta^{2}\right)+u^{2}\left(\alpha^{2}-\beta^{2}\right)\right]}{\left[u^{2}(\alpha-\beta)^{2}+(\gamma-\delta)^{2}\right]\left[u^{2}(\alpha+\beta)^{2}+(\gamma+\delta)^{2}\right]} \\
C & =-A\left(u^{2} \beta^{2}+\delta^{2}\right) \\
D & =\frac{(\beta \gamma-\alpha \delta)\left[u^{4} \alpha \beta\left(\beta^{2}-\alpha^{2}\right)+u^{2}(\beta \gamma-\alpha \delta)(3 \alpha \gamma-\beta \delta)+\gamma \delta\left(\gamma^{2}-\delta^{2}\right)\right]}{\left[u^{2}(\alpha-\beta)^{2}+(\gamma-\delta)^{2}\right]\left[u^{2}(\alpha+\beta)^{2}+(\gamma+\delta)^{2}\right]} .
\end{aligned}
$$

We start by evaluating the first part of the integral (2.2):

$$
\begin{aligned}
\int_{-\infty}^{\infty} & {\left[\frac{A v}{1+v^{2}}+\frac{C v}{v^{2}\left(u^{2} \beta^{2}+\delta^{2}\right)+2 v u(\beta \gamma-\delta \alpha)+u^{2} \alpha^{2}+\gamma^{2}}\right] \mathrm{d} v } \\
= & \frac{A}{2} \int_{-\infty}^{\infty}\left[\frac{2 v}{1+v^{2}}-\frac{2 v\left(u^{2} \beta^{2}+\delta^{2}\right) \pm 2 u(\beta \gamma-\delta \alpha)}{v^{2}\left(u^{2} \beta^{2}+\delta^{2}\right)+2 v u(\beta \gamma-\delta \alpha)+u^{2} \alpha^{2}+\gamma^{2}}\right] \mathrm{d} v \\
= & \left.\frac{A}{2} \lim _{d \rightarrow \infty, c \rightarrow-\infty} \log \left(\frac{1+v^{2}}{v^{2}\left(u^{2} \beta^{2}+\delta^{2}\right)+2 v u(\beta \gamma-\delta \alpha)+u^{2} \alpha^{2}+\gamma^{2}}\right)\right|_{c} ^{d} \\
& +A u(\beta \gamma-\delta \alpha) \int_{-\infty}^{\infty} \frac{1}{v^{2}\left(u^{2} \beta^{2}+\delta^{2}\right)+2 v u(\beta \gamma-\delta \alpha)+u^{2} \alpha^{2}+\gamma^{2}} \mathrm{~d} v \\
= & A u(\beta \gamma-\delta \alpha) \int_{-\infty}^{\infty} \frac{1}{v^{2}\left(u^{2} \beta^{2}+\delta^{2}\right)+2 v u(\beta \gamma-\delta \alpha)+u^{2} \alpha^{2}+\gamma^{2}} \mathrm{~d} v \\
= & A u(\beta \gamma-\delta \alpha) \frac{\pi}{u^{2} \alpha \beta+\gamma \delta},
\end{aligned}
$$

where the last integral is obtained by means of the change of variable

$v \sqrt{u^{2} \beta^{2}+\delta^{2}}+\frac{u(\beta \gamma-\delta \alpha)}{\sqrt{u^{2} \beta^{2}+\delta^{2}}}=z \sqrt{u^{2} \alpha^{2}+\gamma^{2}-\frac{u^{2}(\beta \gamma-\delta \alpha)^{2}}{u^{2} \beta^{2}+\delta^{2}}}=z \frac{u^{2} \alpha \beta+\gamma \delta}{\sqrt{u^{2} \beta^{2}+\delta^{2}}}$.

In view of result (2.3) and inserting the values of $A, B$ and $D$ we have that

$$
\begin{aligned}
\frac{\mathrm{d} u}{\pi^{2}} \int_{-\infty}^{\infty}\left[\frac{A v+B}{1+v^{2}}+\frac{C v+D}{v^{2}\left(u^{2} \beta^{2}+\delta^{2}\right)+2 v u(\beta \gamma-\delta \alpha)+u^{2} \alpha^{2}+\gamma^{2}}\right] \mathrm{d} v \\
=\frac{\mathrm{d} u}{\pi} \frac{1}{u^{2} \alpha \beta+\gamma \delta}\left[A u(\beta \gamma-\alpha \delta)+B\left(u^{2} \alpha \beta+\gamma \delta\right)+D\right] \\
=\frac{\mathrm{d} u}{\pi}\left[\frac{\left(\gamma \delta+u^{2} \alpha \beta\right)(\beta \gamma-\alpha \delta)\left[u^{2}\left(\beta^{2}-\alpha^{2}\right)+\left(\gamma^{2}-\delta^{2}\right)\right]}{\left(u^{2} \alpha \beta+\gamma \delta\right)\left[u^{2}(\alpha-\beta)^{2}+(\gamma-\delta)^{2}\right]\left[u^{2}(\alpha+\beta)^{2}+(\gamma+\delta)^{2}\right]}\right. \\
\left.\quad+\frac{\left(\gamma \delta+u^{2} \alpha \beta\right)(\alpha \gamma-\beta \delta)\left[u^{2}\left(\alpha^{2}-\beta^{2}\right)+\left(\gamma^{2}-\delta^{2}\right)\right]}{\left(u^{2} \alpha \beta+\gamma \delta\right)\left[u^{2}(\alpha-\beta)^{2}+(\gamma-\delta)^{2}\right]\left[u^{2}(\alpha+\beta)^{2}+(\gamma+\delta)^{2}\right]}\right] \\
=\frac{\mathrm{d} u}{\pi} \frac{u^{2}\left(\beta^{2}-\alpha^{2}\right)(\beta-\alpha)(\gamma+\delta)+\left(\gamma^{2}-\delta^{2}\right)(\alpha+\beta)(\gamma-\delta)}{\left[u^{2}(\alpha-\beta)^{2}+(\gamma-\delta)^{2}\right]\left[u^{2}(\alpha+\beta)^{2}+(\gamma+\delta)^{2}\right]} \\
=\frac{\mathrm{d} u}{\pi} \frac{(\alpha+\beta)(\gamma+\delta)\left[u^{2}(\alpha-\beta)^{2}+(\gamma-\delta)^{2}\right]}{\left[u^{2}(\alpha-\beta)^{2}+(\gamma-\delta)^{2}\right]\left[u^{2}(\alpha+\beta)^{2}+(\gamma+\delta)^{2}\right]}
\end{aligned}
$$




$$
=\frac{\mathrm{d} u}{\pi} \frac{(\alpha+\beta)(\gamma+\delta)}{u^{2}(\alpha+\beta)^{2}+(\gamma+\delta)^{2}} .
$$

Another approach is based on the conditional characteristic function,

$$
\begin{aligned}
\mathbf{E}\left\{e^{i \beta U} \mid \mathbf{C}_{2}=v\right\}=\frac{1}{\pi} \int_{-\infty}^{\infty} e^{i \beta(\gamma u+\delta v) /(\alpha-\beta u v)} \frac{\mathrm{d} u}{1+u^{2}} \\
=\frac{1}{\pi} \int_{-\infty}^{\infty} e^{i \beta w} \frac{\alpha \gamma+\delta \beta v^{2}}{v^{2}\left(\beta^{2} w^{2}+\delta^{2}\right)+2 v w(\beta \gamma-\delta \alpha)+w^{2} \alpha^{2}+\gamma^{2}} \mathrm{~d} w,
\end{aligned}
$$

where $w=(\gamma u+\delta v) /(\alpha-\beta u v)$. The inverse Fourier transform gives the conditional density $g(w \mid v)$ and thus we arrive again at the integral (2.2). Theorem 2.1 is proved.

$\mathrm{R}$ e $\mathrm{m}$ a r k 2.1. A special case implied by Theorem 2.1 concerns the random variable

$$
V=\frac{C_{1}+C_{2}}{1-C_{1} C_{2}}
$$

where $C_{j} \sim C_{\left(a_{j}, 0\right)}, j=1,2$, are independent Cauchy random variables with location parameter equal to zero and scale parameter $a_{j}>0$. If we choose $\gamma=a_{1}, \delta=a_{2}, \alpha=1$, and $\beta=a_{1} a_{2}$, we conclude that

$$
V \stackrel{\text { law }}{=} \frac{a_{1} \mathbf{C}_{1}+a_{2} \mathbf{C}_{1}}{1-a_{1} a_{2} \mathbf{C}_{1} \mathbf{C}_{2}} \stackrel{\text { law }}{=} \frac{a_{1}+a_{2}}{1+a_{1} a_{2}} \mathbf{C}
$$

In the same way we obtain that

$$
\widehat{V}=\frac{\gamma C_{1}+\delta C_{2}}{\alpha-\beta C_{1} C_{2}} \stackrel{\text { law }}{=} \frac{\gamma a_{1} \mathbf{C}_{1}+\delta a_{2} \mathbf{C}_{2}}{\alpha-\beta a_{1} a_{2} \mathbf{C}_{1} \mathbf{C}_{2}} \stackrel{\text { law }}{=} \frac{\gamma a_{1}+\delta a_{2}}{\alpha+\beta a_{1} a_{2}} \mathbf{C}
$$

where $\alpha+\beta a_{1} a_{2} \neq 0$.

$\mathrm{R}$ e $\mathrm{m}$ a $\mathrm{rk}$ 2.2. In view of Theorem 2.1 we can obtain by recurrence the distribution of the current angle after $n$ steps for the angular random walk

$$
S_{n}=\sum_{j=1}^{n} \operatorname{arctg} C_{j}
$$

described in the introduction. We have

$$
S_{n}=\sum_{j=1}^{n} \operatorname{arctg} C_{\left(a_{j}, 0\right)}=\operatorname{arctg} C_{\left(\hat{a}_{n-1}, 0\right)}+\operatorname{arctg} C_{\left(a_{n}, 0\right)},
$$

where $\operatorname{arctg} C_{\left(\hat{a}_{n-1}, 0\right)}$ is the random variable $S_{n-1}$. In particular, if $a_{j}=1$ for $j=1, \ldots, n$, we have the following property of the standard Cauchy random variables $\mathbf{C}_{j}$ :

$$
\sum_{j=1}^{n} \operatorname{arctg} \mathbf{C}_{j} \stackrel{\operatorname{law}}{=} \operatorname{arctg} \mathbf{C}
$$


$\mathrm{R}$ e $\mathrm{m}$ a $\mathrm{r} \mathrm{k} 2.3$. A simple byproduct of Theorem 2.1 is that

$$
\begin{aligned}
\mathbf{E} e^{i \beta U} & =\frac{1}{\pi^{2}} \int_{\mathbf{R}^{2}} \exp \left\{i \beta \frac{x+y}{1-x y}\right\} \frac{a_{1} a_{2} \mathrm{~d} x \mathrm{~d} y}{\left(a_{1}^{2}+x^{2}\right)\left(a_{2}^{2}+y^{2}\right)} \\
& =\frac{1}{\pi^{2}} \int_{-\pi / 2}^{\pi / 2} \int_{-\pi / 2}^{\pi / 2} \exp \left\{i \beta \frac{a_{1} \operatorname{tg} \theta_{1}+a_{2} \operatorname{tg} \theta_{2}}{1-a_{1} a_{2} \operatorname{tg} \theta_{1} \operatorname{tg} \theta_{2}}\right\} \mathrm{d} \theta_{1} \mathrm{~d} \theta_{2} \\
& =\exp \left\{-\frac{a_{1}+a_{2}}{1+a_{1} a_{2}}|\beta|\right\} .
\end{aligned}
$$

In (2.4) we have used the transformations $x=a_{1} \operatorname{tg} \theta_{1}$ and $y=a_{2} \operatorname{tg} \theta_{2}$. In the special case $a_{1}=a_{2}=1$ the relationship (2.4) yields

$$
\begin{aligned}
e^{-|\beta|} & =\frac{1}{\pi^{2}} \int_{-\pi / 2}^{\pi / 2} \int_{-\pi / 2}^{\pi / 2} \exp \left\{i \beta \frac{\operatorname{tg} \theta_{1}+\operatorname{tg} \theta_{2}}{1-\operatorname{tg} \theta_{1} \operatorname{tg} \theta_{2}}\right\} \mathrm{d} \theta_{1} \mathrm{~d} \theta_{2} \\
& =\frac{1}{\pi^{2}} \int_{-\pi / 2}^{\pi / 2} \int_{-\pi / 2}^{\pi / 2} e^{i \beta \operatorname{tg}\left(\theta_{1}+\theta_{2}\right)} \mathrm{d} \theta_{1} \mathrm{~d} \theta_{2}=\frac{2}{\pi^{2}} \int_{0}^{\pi} x \cos (\beta \operatorname{tg} x) \mathrm{d} x .
\end{aligned}
$$

In the last step of (2.5) we have used the transformations $\theta_{1}+\theta_{2}=x$ and $\theta_{2}=y$. The integral (2.5) shows that, if $\left(\Theta_{1}, \Theta_{2}\right)$ is uniform in the square $S=\left\{\left(\theta_{1}, \theta_{2}\right):-\pi / 2<\left|\theta_{i}\right|<\pi / 2, i=1,2\right\}$, then the random variable $W=\operatorname{tg}\left(\Theta_{1}+\Theta_{2}\right)$ has characteristic function $e^{-|\beta|}$ because $\Theta_{1}+\Theta_{2}$ is uniform and therefore $W$ is Cauchy distributed.

$\mathrm{R}$ e $\mathrm{m}$ a r k 2.4. It is well known that for a planar Brownian motion $\left\{\left(B_{1}(t), B_{2}(t)\right), t>0\right\}$ starting from $(x, y)$ the random variable $B_{1}\left(T_{y}\right)$ possesses Cauchy distribution with parameters $(x, y)$, where

$$
T_{y}=\inf \left\{t>0: B_{2}(t)=0\right\} .
$$

If the starting points of two planar Brownian motions $\left(B_{1}^{i}(t), B_{2}^{i}(t)\right)$, for $i=1,2$, are located on the $y$ axis as in Figure 4, then we have that

$$
\begin{aligned}
\Theta=\Theta_{1}+\Theta_{2} & =\operatorname{arctg} B_{1}^{1}\left(T_{a_{1}}\right)+\operatorname{arctg} B_{1}^{2}\left(T_{a_{2}}\right) \\
& \stackrel{\text { law }}{=} \operatorname{arctg} C_{1}+\operatorname{arctg} C_{2} \stackrel{\text { law }}{=} \operatorname{arctg} \frac{a_{1}+a_{2}}{1+a_{1} a_{2}} \mathbf{C},
\end{aligned}
$$

where $C_{1}$ and $C_{2}$ are two independent Cauchy random variables with scale parameters $a_{1}$ and $a_{2}$, respectively, and location parameters equal to zero. Therefore if the starting point of a third Brownian motion has coordinates $\left(0,\left(a_{1}+a_{2}\right) /\left(1+a_{1} a_{2}\right)\right)$ then $B\left(T_{\left(a_{1}+a_{2}\right) /\left(1+a_{1} a_{2}\right)}\right)$ represents its hitting position on the $x$-axis. This point forms with $(0,1)$ and the origin a right triangle with an angle equal to $\Theta=\Theta_{1}+\Theta_{2}$. 

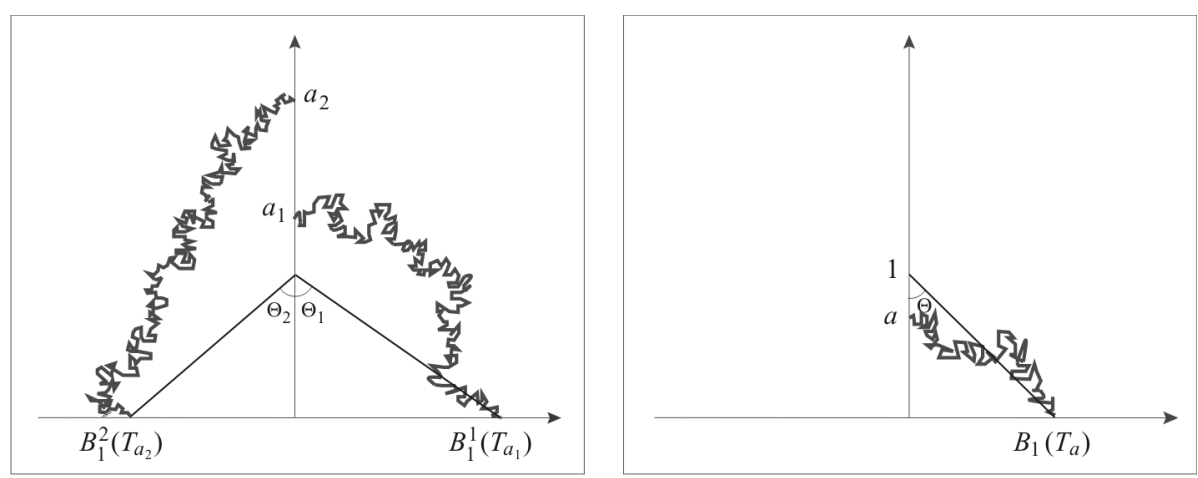

Fig. 4. The hitting position on the $x$-axis of a planar Brownian motion is Cauchy distributed. In the figure the random angles $\Theta_{1}, \Theta_{2}$, and $\Theta=\Theta_{1}+\Theta_{2}$ are shown. For $a=\left(a_{1}+a_{2}\right) /\left(1+a_{1} a_{2}\right)$ the right-hand figure shows the hitting position $B_{1}\left(T_{a}\right)$

3. Noncentered Cauchy random variables. For independent Cauchy random variables $C_{1}$ and $C_{2}$, with location parameters $b_{1}$ and $b_{2}$ and scale parameters $a_{1}$ and $a_{2}$, the random variable $U$ in (2.1) is still Cauchy distributed with both parameters affected by the values of the location parameters $b_{1}, b_{2}$ and the scale parameters $a_{1}, a_{2}$.

Theorem 3.1. If $C_{i}, i=1,2$, are two independent Cauchy random variables with location parameters $b_{i}$ and scale parameters $a_{i}$, then the random variable $U$ is still Cauchy distributed with scale parameter

$$
a_{U}=\frac{\left(a_{1}+a_{2}\right)\left(1+a_{1} a_{2}-b_{1} b_{2}\right)+\left(b_{1}+b_{2}\right)\left(a_{1} b_{2}+a_{2} b_{1}\right)}{\left(1+a_{1} a_{2}-b_{1} b_{2}\right)^{2}+\left(a_{1} b_{2}+a_{2} b_{1}\right)^{2}}
$$

and location parameter

$$
b_{U}=\frac{\left(a_{1}+a_{2}\right)\left(a_{1} b_{2}+a_{2} b_{1}\right)-\left(b_{1}+b_{2}\right)\left(1+a_{1} a_{2}-b_{1} b_{2}\right)}{\left(1+a_{1} a_{2}-b_{1} b_{2}\right)^{2}+\left(a_{1} b_{2}+a_{2} b_{1}\right)^{2}} .
$$

P r o o f. We obtain the density function of the random variable $U$ by observing that

$$
\mathbf{P}\{U \in \mathrm{d} w\}=\mathbf{E}\left\{\mathbf{P}\left\{\frac{C_{1}+C_{2}}{1-C_{1} C_{2}} \in \mathrm{d} w \mid C_{2}\right\}\right\}
$$

and remarking that

$$
\mathbf{P}\left\{\frac{C_{1}+C_{2}}{1-C_{1} C_{2}} \in \mathrm{d} w \mid C_{2}=y\right\}=\frac{a_{1}}{\pi\left[(w-y) /(1+w y)-b_{1}\right]^{2}+a_{1}^{2}} \frac{1+y^{2}}{(1+w y)^{2}} \mathrm{~d} w .
$$


Therefore

$$
\begin{aligned}
\mathbf{P} & \{U \in \mathrm{d} w\} \\
& =\frac{a_{1} a_{2} \mathrm{~d} w}{\pi^{2}} \int_{-\infty}^{\infty} \frac{1}{\left[(w-y) /(1+w y)-b_{1}\right]^{2}+a_{1}^{2}} \frac{1+y^{2}}{(1+w y)^{2}} \frac{1}{\left(y-b_{2}\right)^{2}+a_{2}^{2}} \mathrm{~d} y \\
& =\frac{a_{1} a_{2} \mathrm{~d} w}{\pi^{2}} \int_{-\infty}^{\infty} \frac{1+y^{2}}{\left[w-y-b_{1}(1+w y)\right]^{2}+a_{1}^{2}(1+w y)^{2}} \frac{1}{\left(y-b_{2}\right)^{2}+a_{2}^{2}} \mathrm{~d} y \\
& =\frac{a_{1} a_{2} \mathrm{~d} w}{\pi^{2}} \int_{-\infty}^{\infty} \frac{1+y^{2}}{\left[y^{2} E-2 y F+G\right]\left[y^{2}-2 y H+K\right]} \mathrm{d} y
\end{aligned}
$$

where

$$
\begin{gathered}
E=1+2 b_{1} w+\left(a_{1}^{2}+b_{1}^{2}\right) w^{2}, \quad F=-b_{1}-\left(a_{1}^{2}+b_{1}^{2}-1\right) w+b_{1} w^{2}, \\
G=a_{1}^{2}+b_{1}^{2}-2 b_{1} w+w^{2}, \quad H=b_{2}, \quad K=a_{2}^{2}+b_{2}^{2} .
\end{gathered}
$$

We rewrite the integral in (3.1) in the following form:

$$
\mathbf{P}\{U \in \mathrm{d} w\}=\frac{a_{1} a_{2} \mathrm{~d} w}{\pi^{2}} \int_{-\infty}^{\infty}\left[\frac{A y+B}{y^{2} E-2 y F+G}+\frac{C y+D}{y^{2}-2 y H+K}\right] \mathrm{d} y
$$

with

$$
\begin{gathered}
A+C E=0, \quad-2 A H+B-2 C F+D E=1, \\
A K-2 H B+C G-2 D F=0, \quad B K+D G=1 .
\end{gathered}
$$

The integrals in (3.2) can be worked out by means of the changes of variables

$$
y \sqrt{E}-\frac{F}{\sqrt{E}}=x \sqrt{G-\frac{F^{2}}{E}}, \quad y-H=x \sqrt{K-H^{2}} .
$$

The first integral becomes

$$
\begin{aligned}
\int_{-\infty}^{\infty} & \frac{A y+B}{(y \sqrt{E}-F / \sqrt{E})^{2}+G-F^{2} / E} \mathrm{~d} y \\
& =\int_{-\infty}^{\infty} \frac{1}{E} \frac{B E+A F+x A \sqrt{G E-F^{2}}}{\left(x^{2}+1\right) \sqrt{G E-F^{2}}} \mathrm{~d} x
\end{aligned}
$$

and the second one takes the form

$$
\int_{-\infty}^{\infty} \frac{C y+D}{(y-H)^{2}+K-H^{2}} \mathrm{~d} y=\int_{-\infty}^{\infty} \frac{D+C H+x C \sqrt{K-H^{2}}}{\left(x^{2}+1\right) \sqrt{K-H^{2}}} \mathrm{~d} x .
$$

A substantial simplification can be obtained because

$$
\sqrt{G E-F^{2}}=a_{1}\left(1+w^{2}\right), \quad \sqrt{H-K^{2}}=a_{2} .
$$


If we turn back to the distribution (3.2), in view of the above calculations, we have that

$$
\begin{aligned}
\mathbf{P}\{U \in \mathrm{d} w\}= & \frac{a_{2} \mathrm{~d} w}{\pi^{2}\left(1+w^{2}\right) E} \int_{-\infty}^{\infty} \frac{B E+A F+x A a_{1}\left(1+w^{2}\right)}{x^{2}+1} \mathrm{~d} x \\
& +\frac{a_{1} \mathrm{~d} w}{\pi^{2}} \int_{-\infty}^{\infty} \frac{D+C H+x C a_{2}}{1+x^{2}} \mathrm{~d} x
\end{aligned}
$$

We observe that, by the first equation of (3.3),

$$
\frac{a_{1} a_{2} \mathrm{~d} w}{\pi^{2}}\left(\frac{A}{E}+C\right) \int_{-\infty}^{\infty} \frac{x}{1+x^{2}} \mathrm{~d} x=0
$$

and

$$
\begin{aligned}
\mathbf{P}\{U \in \mathrm{d} w\} & =\left\{a_{2} \frac{B E+A F}{\pi E\left(1+w^{2}\right)}+a_{1} \frac{D+C H}{\pi}\right\} \mathrm{d} w \\
& =\left\{a_{2} \frac{B-C F}{\pi\left(1+w^{2}\right)}+a_{1} \frac{D+C H}{\pi}\right\} \mathrm{d} w .
\end{aligned}
$$

The values of $B, C$, and $D$ can be derived by solving the system (3.3), simplified as

$$
\begin{gathered}
B+2 C(H E-F)+D E=1, \quad-2 B H+C(G-E K)-2 D F=0, \\
B K+D G=1 .
\end{gathered}
$$

By means of cumbersome computations we arrive at the final result

$$
\begin{aligned}
& \mathbf{P}\{U \in \mathrm{d} w\} \\
&=\frac{1}{\pi}\left\{\left(1+a_{1} a_{2}-b_{1} b_{2}\right)\left[w\left(a_{1} b_{2}+a_{2} b_{1}\right)+a_{1}+a_{2}\right]\right. \\
&\left.\quad-\left(a_{1} b_{2}+a_{2} b_{1}\right)\left[w\left(1+a_{1} a_{2}-b_{1} b_{2}\right)-\left(b_{1}+b_{2}\right)\right]\right\} \\
& \quad \times\left\{\left[w\left(1+a_{1} a_{2}-b_{1} b_{2}\right)-\left(b_{1}+b_{2}\right)\right]^{2}+\left[w\left(a_{1} b_{2}+a_{2} b_{1}\right)+a_{1}+a_{2}\right]^{2}\right\}^{-1} \\
&=\frac{\left(a_{1}+a_{2}\right)\left(1+a_{1} a_{2}-b_{1} b_{2}\right)+\left(b_{1}+b_{2}\right)\left(a_{1} b_{2}+a_{2} b_{1}\right)}{\left(1+a_{1} a_{2}-b_{1} b_{2}\right)^{2}+\left(a_{1} b_{2}+a_{2} b_{1}\right)^{2}} \\
& \quad \times\left\{\left[w+\frac{\left(a_{1}+a_{2}\right)\left(a_{1} b_{2}+a_{2} b_{1}\right)-\left(b_{1}+b_{2}\right)\left(1+a_{1} a_{2}-b_{1} b_{2}\right)}{\left(1+a_{1} a_{2}-b_{1} b_{2}\right)^{2}+\left(a_{1} b_{2}+a_{2} b_{1}\right)^{2}}\right]^{2}\right. \\
&\left.\quad+\left[\frac{\left(a_{1}+a_{2}\right)\left(1+a_{1} a_{2}-b_{1} b_{2}\right)+\left(b_{1}+b_{2}\right)\left(a_{1} b_{2}+a_{2} b_{1}\right)}{\left(1+a_{1} a_{2}-b_{1} b_{2}\right)^{2}+\left(a_{1} b_{2}+a_{2} b_{1}\right)^{2}}\right]^{2}\right\}^{-1} .
\end{aligned}
$$

Theorem 3.1 is proved.

$\mathrm{R} \mathrm{e} \mathrm{m}$ a $\mathrm{r} \mathrm{k}$ 3.1. In view of Theorem 3.1 it is possible to obtain the following particular cases. 
(i) For $a_{1}=a_{2}=1$ and $b_{1}=b_{2}=b$, we have that

$$
a_{U}=\frac{2 b^{2}+4}{b^{4}+4}, \quad b_{U}=\frac{2 b^{3}}{b^{4}+4} .
$$

This shows that $U$ has center of symmetry on the positive half-line if $b>0$ and on the negative half-line if $b<0$, therefore the nonlinear transformation $U$ preserves the sign of the mode.

(ii) For $a_{1}=a_{2}=a$ and $b_{1}=b_{2}=b$ we have that

$$
a_{U}=\frac{2 a\left(1+a^{2}+b^{2}\right)}{\left(1+a^{2}-b^{2}\right)^{2}+(2 a b)^{2}}, \quad b_{U}=\frac{2 b\left(a^{2}+b^{2}-1\right)}{\left(1+a^{2}-b^{2}\right)^{2}+(2 a b)^{2}} .
$$

We note that $a_{U}$ and $b_{U}$ depend simultaneously from the scale and location parameters of the random variables involved in $U$.

4. Continued fractions. The property that the reciprocal of a Cauchy random variable has still a Cauchy distribution has a number of possible extensions which we deal with in this section.

We start by considering the sequence

$$
V_{1}=\frac{1}{1+\mathbf{C}}, \quad V_{2}=\frac{1}{1+\frac{1}{1+\mathbf{C}}}, \quad \ldots, \quad V_{n}=\frac{1}{1+\frac{1}{1+\cdots+\frac{1}{1+\mathbf{C}}}},
$$

and prove the following theorem.

Theorem 4.1. The random variables defined in (4.1) have Cauchy distribution, $V_{n} \sim C_{\left(a_{n}, b_{n}\right)}$, where the scale parameters $a_{n}$ and the location parameters $b_{n}$ satisfy the recursive relationships

$$
\begin{array}{ll}
a_{n+1}=\frac{a_{n}}{\left(1+b_{n}\right)^{2}+a_{n}^{2}}, & n=1,2, \ldots, \\
b_{n+1}=\frac{b_{n}+1}{\left(1+b_{n}\right)^{2}+a_{n}^{2}}, & n=1,2, \ldots
\end{array}
$$

P r o o f. Let us assume that $V_{n}$ possesses Cauchy density with parameters $a_{n}$ and $b_{n}$. Then $V_{n+1}$ writes

$$
\mathbf{P}\left\{V_{n+1}<v\right\}=\mathbf{P}\left\{\frac{1}{1+V_{n}}<v\right\}=\mathbf{P}\left\{\frac{1}{1+a_{n}+b_{n} \mathbf{C}}<v\right\} .
$$

After some computations the density of $V_{n+1}$ can be written as

$$
f_{V_{n+1}}(v)=\frac{a_{n} /\left(\left(1+b_{n}\right)^{2}+a_{n}^{2}\right)}{\pi\left[v-\left(b_{n}+1\right) /\left(\left(1+b_{n}\right)^{2}+a_{n}^{2}\right)\right]^{2}+a_{n}^{2} /\left[\left(1+b_{n}\right)^{2}+a_{n}^{2}\right]^{2}}, \quad v \in \mathbf{R} .
$$

It can be ascertained directly that $V_{1}$ possesses Cauchy distribution with parameters $a_{1}=1 / 2$ and $b_{1}=1 / 2$.

Theorem 4.1 is proved.

$\mathrm{R} \mathrm{e} \mathrm{m}$ a r $\mathrm{k}$ 4.1. We have evaluated the following table of parameters $a_{n}$ and $b_{n}$ : 


\begin{tabular}{|c|c|c|c|c|c|}
\hline$n$ & 1 & 2 & 3 & $\ldots$ & $10^{2}$ \\
\hline$a_{n}$ & $1 / 2$ & $1 / 5$ & $1 / 13$ & $\ldots$ & $5.77 e^{-42}$ \\
\hline$b_{n}$ & $1 / 2$ & $3 / 5$ & $8 / 13$ & $\ldots$ & 0.618034 \\
\hline
\end{tabular}

For $n=1,2,3$ we can observe that the scale parameters $a_{n}$ coincide with the inverse of the odd-indexed Fibonacci numbers while the sequence $b_{n}$ has the numerators coinciding with the even-indexed Fibonacci numbers and the denominators correspond to the odd-indexed Fibonacci numbers.

In light of the previous considerations we can show that for $n \geqslant 1$

$$
b_{n}=\frac{F_{2 n}}{F_{2 n+1}}, \quad a_{n}=\frac{1}{F_{2 n+1}},
$$

where $F_{n}, n \geqslant 0$, is the Fibonacci sequence. Recalling that the Fibonacci numbers admit the representation (it can easily be checked by induction)

$$
F_{n}=\frac{\varphi^{n}-(1-\varphi)^{n}}{\sqrt{5}},
$$

where $\varphi=(1+\sqrt{5}) / 2$ is the golden ratio, we now prove that if $a_{n}$ and $b_{n}$ have the representations (4.4), then also $a_{n+1}$ and $b_{n+1}$ can be expressed in the same form. From (4.2) and (4.3) we have

$$
\begin{aligned}
b_{n+1} & =\frac{F_{2 n} / F_{2 n+1}+1}{\left(F_{2 n} / F_{2 n+1}+1\right)^{2}+1 / F_{2 n+1}^{2}}=\frac{F_{2 n+2} F_{2 n+1}}{F_{2 n+2}^{2}+1} \\
& =F_{2 n+2}\left[\frac{\varphi^{2 n+1}-(1-\varphi)^{2 n+1}}{\varphi^{4 n+4}+(1-\varphi)^{4 n+4}-2 \varphi^{2 n+2}(1-\varphi)^{2 n+2}+5}\right] \sqrt{5} \\
& =F_{2 n+2}\left[\frac{\varphi^{2 n+1}-(1-\varphi)^{2 n+1}}{\left[\varphi^{2 n+1}-(1-\varphi)^{2 n+1}\right]\left[\varphi^{2 n+3}-(1-\varphi)^{2 n+3}\right]}\right] \sqrt{5}=\frac{F_{2 n+2}}{F_{2 n+3}} .
\end{aligned}
$$

Similar calculations prove that $a_{n+1}=\frac{1}{F_{2 n+3}}$. In view of representations (4.4) and (4.5), it is easy to show that

$$
\begin{aligned}
& \lim _{n \rightarrow \infty} b_{n}=\lim _{n \rightarrow \infty} \frac{F_{2 n}}{F_{2 n+1}}=\lim _{n \rightarrow \infty} \frac{1-((1-\varphi) / \varphi)^{2 n}}{\varphi-(1-\varphi)((1-\varphi) / \varphi)^{2 n}}=\frac{1}{\varphi}=\varphi-1, \\
& \lim _{n \rightarrow \infty} a_{n}=\lim _{n \rightarrow \infty} \frac{1}{F_{2 n+1}}=0 .
\end{aligned}
$$

Otherwise, observing that the sequence $b_{n}, n \geqslant 1$, is increasing, because

$$
\frac{b_{n+1}}{b_{n}}=\frac{F_{2 n+2} F_{2 n+1}}{F_{2 n+3} F_{2 n}}=\frac{\varphi^{4 n+3}+(1-\varphi)^{4 n+3}+1}{\varphi^{4 n+3}+(1-\varphi)^{4 n+3}-4} \geqslant 1,
$$

and taking the limits in (4.2) and (4.3) we have that

$$
L=\frac{L}{(1+H)^{2}+L^{2}}, \quad H=\frac{H+1}{(1+H)^{2}+L^{2}},
$$


where $H=\lim _{n \rightarrow \infty} a_{n}$ and $L=\lim _{n \rightarrow \infty} b_{n}$. From the relationships (4.6) we derive the equality

$$
\frac{L}{H}=\frac{L}{H+1}
$$

that implies $L=0$. In fact, for $L \neq 0$, we arrive at the absurd that $H=$ $H+1$. Substituting $L=0$ in the second formula of (4.6) we obtain

$$
H=\frac{1}{H+1} \text {. }
$$

Since $H$ satisfies the algebraic equation $H^{2}+H-1=0$, it follows that $H=\varphi-1$, where $\varphi$ is the golden ratio (see Figure 5).
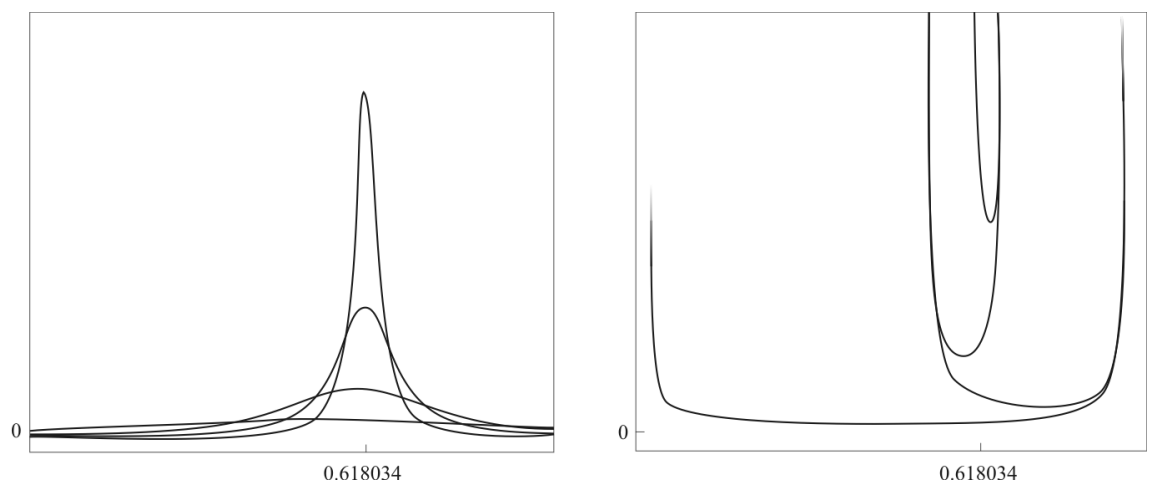

Fig. 5. In the left figure the densities of the Cauchy random variables $V_{1}, V_{2}, V_{3}$, and $V_{4}$ are shown. In the right figure the densities of $U_{1}, U_{2}, U_{3}$ and $U_{4}$ are plotted

$\mathrm{R}$ e $\mathrm{m}$ a $\mathrm{r} \mathrm{k}$ 4.2. A slightly more general case concerns the sequence

$$
\begin{aligned}
& W_{1}=\frac{1}{c_{1}+d_{1} C_{\left(a_{0}, b_{0}\right)}}=\frac{1}{c_{1}+a_{0} d_{1}+b_{0} d_{1} \mathbf{C}}, \\
& W_{2}=\frac{1}{c_{2}+d_{2} W_{1}}, \quad W_{3}=\frac{1}{c_{3}+d_{3} W_{2}}, \ldots
\end{aligned}
$$

By performing calculations similar to those of Theorem 4.1 we have that $W_{1}$ has Cauchy distribution with scale parameter $a_{1}$ and location parameter $b_{1}$ such that

$$
a_{1}=\frac{d_{1} a_{0}}{\left(c_{1}+d_{1} b_{0}\right)^{2}+d_{1}^{2} a_{0}^{2}}, \quad b_{1}=\frac{c_{1}+d_{1} b_{0}}{\left(c_{1}+d_{1} b_{0}\right)^{2}+d_{1}^{2} a_{0}^{2}} .
$$

Similarly, if $W_{n} \sim C_{\left(a_{n}, b_{n}\right)}$, then $W_{n+1} \sim C_{\left(a_{n+1}, b_{n+1}\right)}$, where

$$
\begin{aligned}
a_{n+1} & =\frac{d_{n+1} a_{n}}{\left(c_{n+1}+d_{n+1} b_{n}\right)^{2}+d_{n+1}^{2} a_{n}^{2}}, \\
b_{n+1} & =\frac{c_{n+1}+d_{n+1} b_{n}}{\left(c_{n+1}+d_{n+1} b_{n}\right)^{2}+d_{n+1}^{2} a_{n}^{2}}
\end{aligned}
$$


for every $n \geqslant 2$. The sequences in (4.7) for $c_{n}=d_{n}=1$ coincide with (4.2) and (4.3).

Another sequence of continued fractions involving the Cauchy distribution is the following one:

$$
U_{1}=\frac{1}{1+\mathbf{C}^{2}}, \quad U_{2}=\frac{1}{1+\frac{1}{1+\mathbf{C}^{2}}}, \quad \ldots, \quad U_{n}=\frac{1}{1+\frac{1}{1+\cdots+\frac{1}{1+\mathbf{C}^{2}}}} .
$$

It is well known that the random variable $U_{1}$ possesses the arcsine law. Unlike the sequence $V_{n}$ studied above the sequence $U_{n}, n \geqslant 1$, has a density structure changing with $n$. Some calculations are sufficient to show that $U_{1}, U_{2}, U_{3}, U_{4}$ have density, respectively, equal to

$$
\begin{aligned}
& f_{U_{1}}(u)=\frac{1}{\pi \sqrt{u(1-u)}, \quad 0<u<1,}, \quad \frac{1}{2}<u<1, \\
& f_{U_{2}}(u)=\frac{1}{\pi u \sqrt{(1-u)(2 u-1)},}, \quad \frac{1}{2}<u<\frac{2}{3}, \\
& f_{U_{3}}(u)=\frac{1}{\pi(1-u) \sqrt{(2 u-1)(2-3 u)}}, \quad \frac{3}{5}<u<\frac{2}{3} . \\
& f_{U_{4}}(u)=\frac{1}{\pi(2 u-1) \sqrt{(2-3 u)(5 u-3)}}, \quad
\end{aligned}
$$

The general result concerning $U_{n}$ is stated in the following theorem.

Theorem 4.2. For every $n \geqslant 1$ the distribution of the random variable $U_{n}$ is given by

$$
\begin{aligned}
\mathbf{P}\left\{U_{n} \in \mathrm{d} u\right\}= & \frac{1}{\pi\left[(-1)^{n+1} \alpha_{n}+(-1)^{n} \beta_{n} u\right]} \frac{1}{\sqrt{(-1)^{n} \beta_{n}+(-1)^{n+1}\left(\alpha_{n}+\beta_{n}\right) u}} \\
& \times \frac{1}{\sqrt{(-1)^{n+1}\left(\alpha_{n}+\beta_{n}\right)+(-1)^{n}\left(\alpha_{n}+2 \beta_{n}\right) u}} \mathrm{~d} u
\end{aligned}
$$

where $(-1)^{n}\left(\alpha_{n}+\beta_{n}\right) /\left(\alpha_{n}+2 \beta_{n}\right)<(-1)^{n} u<(-1)^{n} \beta_{n} /\left(\alpha_{n}+\beta_{n}\right)$, and $\alpha_{n}, \beta_{n} \in \mathbf{N}$ satisfy the recursive relationships $\alpha_{n}=\beta_{n-1}, \beta_{n}=\alpha_{n-1}+\beta_{n-1}$.

P r o o f. From (4.8) we have that $U_{n+1}=1 /\left(1+U_{n}\right)$. Then proceeding by induction, i.e., assuming that $U_{n}$ has distribution (4.9), we obtain that

$$
\begin{aligned}
\mathbf{P}\left\{U_{n+1} \in \mathrm{d} u\right\}= & \frac{\mathrm{d}}{\mathrm{d} u} \mathbf{P}\left\{U_{n}>\frac{1-u}{u}\right\} \mathrm{d} u=\frac{\mathrm{d}}{\mathrm{d} u} \int_{(1-u) / u}^{h\left(\alpha_{n}, \beta_{n}\right)} \mathbf{P}\left\{U_{n} \in \mathrm{d} u\right\} \\
= & \frac{1}{\pi} \frac{1}{u^{2}} \frac{1}{\left[(-1)^{n+1} \alpha_{n}+(-1)^{n} \beta_{n}(1-u) / u\right]} \\
& \times \frac{1}{\sqrt{(-1)^{n} \beta_{n}+(-1)^{n+1}\left(\alpha_{n}+\beta_{n}\right)(1-u) / u}} \\
& \times \frac{1}{\sqrt{(-1)^{n+1}\left(\alpha_{n}+\beta_{n}\right)+(-1)^{n}\left(\alpha_{n}+2 \beta_{n}\right)(1-u) / u}} \mathrm{~d} u
\end{aligned}
$$




$$
\begin{aligned}
= & \frac{1}{\pi\left[(-1)^{n} \beta_{n}+(-1)^{n+1}\left(\alpha_{n}+\beta_{n}\right) u\right]} \\
& \times \frac{1}{\sqrt{(-1)^{n+1}\left(\alpha_{n}+\beta_{n}\right)+(-1)^{n}\left(\alpha_{n}+2 \beta_{n}\right) u}} \\
& \times \frac{1}{\sqrt{(-1)^{n}\left(\alpha_{n}+2 \beta_{n}\right)+(-1)^{n+1}\left(2 \alpha_{n}+3 \beta_{n}\right) u}} \mathrm{~d} u .
\end{aligned}
$$

In the first integral the function $h\left(\alpha_{n}, \beta_{n}\right)$ represents the right boundary of the support of $U_{n}$. We conclude that $U_{n+1}$ possesses distribution (4.9) by taking $\alpha_{n+1}=\beta_{n}$ and $\beta_{n+1}=\alpha_{n}+\beta_{n}$. Theorem 4.2 is proved.

$\mathrm{R} \mathrm{e} \mathrm{m}$ a r k 4.3. The sequence $\beta_{n}$ is a Fibonacci sequence, since we have that $\beta_{n}=\beta_{n-1}+\alpha_{n-1}=\beta_{n-1}+\beta_{n-2}$. We note that the sequence of coefficients $\alpha_{n}$ and $\beta_{n}$ are such that $\lim _{n \rightarrow \infty} \alpha_{n+1} / \beta_{n+1}=\lim _{n \rightarrow \infty} \beta_{n} / \beta_{n+1}=$ $\varphi-1$. On the base of arguments similar to those of Remark 4.1 it is possible to show that the sequence $U_{n}, n \geqslant 1$, converges in distribution to $\varphi-1$. In this case the upper and lower bounds of the domain of definition of the densities $f_{U_{n}}(u), n \geqslant 1$ are expressed as ratios of Fibonacci numbers (see Figure 5).

Acknowledgment. We are very grateful to the referee for his scholar report and also to have drawn our attention to some relevant references.

\section{REFERENCES}

1. Asci C., Letac G., Piccioni M. Beta-hypergeometric distributions and random continued fractions. - Statist. Probab. Lett., 2008, v. 78, № 13, p. 1711-1721.

2. Cammarota V., Orsingher E. Travelling randomly on the Poincaré half-plane with a Pythagorean compass. - J. Statist. Phys., 2008, v. 130, № 3, p. 455-482.

3. Cammarota V., Orsingher E. Cascades of particles moving at finite velocity in hyperbolic spaces. - J. Statist. Phys., 2008, v. 133, №6, p. 1137-1159.

4. Chamayou J.-F., Letac G. Explicit stationary distributions for compositions of random functions and products of random matrices. - J. Theoret. Probab., 1991, v. 4, № 1, p. 3-36.

5. Faber R. L. Foundations of Euclidean and Non-Euclidean Geometry. New York: Dekker, 1983, 329 p.

6. Knight F. B. A characterization of the Cauchy type. — Proc. Amer. Math. Soc., 1976, v. 55 , № 1 , p. 130-135.

7. Letac G. Which functions preserve Cauchy laws? — Proc. Amer. Math. Soc., 1977, v. 67 , № 2 , p. $277-286$.

8. Pitman E. J. G., Williams E. J. Cauchy-distributed functions of Cauchy variates. Ann. Math. Statist., 1967, v. 38, p. 916-918.

9. Chaumont L., Yor M. Exercises in Probability. Cambridge: Cambridge Univ. Press, 2003, $236 \mathrm{p}$.

10. Williams E. J. Cauchy-distributed functions and a characterization of the Cauchy distribution. - Ann. Math. Statist., 1969, v. 40, № 3, p. 1083-1085.

Поступила в редакцию 10.III.2009

Исправленный вариант 